\title{
Invaginación apendicular*
}

\author{
Drs. CRISTÓBAL SUAZO L. ${ }^{1}$, PATRICIO BURDILES P. ${ }^{1}$, ANDRÉS LARACH K. ${ }^{1}$
}

1 Departamento de Cirugía Adultos, Clínica Las Condes. Santiago, Chile.

\begin{abstract}
\section{Cecal appendix invagination}

Cecal appendix invagination was first described by Mc Kidd in 1858, however, due to its low frequency and nonspecific presentation, it is usually a radiological or intraoperative finding. During surgery, the therapeutic approach may vary from an appendectomy to a right hemicolectomy. The selected technique will depend of the suspected diagnosis, which will undoubtedly also influenced by surgeon experience. We present a case of a male patient aged 57 years old, with the intraoperative finding of appendiceal intussusception. The laparoscopic dissection of the cecum revealed an inflammatory appendix, soft to palpation with graspers. A large section of the appendix base with linear stapler was made. The final biopsy and surgical specimen showed an appendiceal inflammation without atypia.
\end{abstract}

Key words: Appendix, invagination.

\section{Resumen}

La invaginación del apéndice cecal fue descrita por primera vez en 1858, sin embargo, debido a su baja frecuencia y presentación inespecífica suele constituir un hallazgo radiológico o intraoperatorio. En este último caso la conducta terapéutica puede variar desde la apendicectomía hasta la hemicolectomía, ya que se debe plantear a la neoplasia cecal dentro de los diagnósticos diferenciales y, en virtud de ello, la decisión operatoria puede obligar a la hemicolectomía derecha. Esta decisión se verá influida también por la experiencia del cirujano. Se presenta el caso de un paciente de sexo masculino de 57 años de edad con el hallazgo intraoperatorio de invaginación apendicular. Se realizó una disección amplia del ciego por vía laparoscópica y a la palpación con las pinzas el apéndice y el ciego impresionaban de consistencia blanda, no tumoral. Debido a estos hallazgos se decidió realizar la apendicectomía realizando una sección amplia de la base apendicular con stapler lineal cortante. La biopsia definitiva de la pieza operatoria informó un proceso inflamatorio apendicular sin hallazgos sugerentes de atipías.

Palabras clave: Apéndice cecal, invaginación.

*Recibido el 28 de enero de 2011 y aceptado para publicación el 27 de julio de 2011.

Correspondencia: Dr. Patricio Burdiles P.

Lo Fontecilla 441, Santiago, Chile.

pburdiles@clc.cl 


\section{Caso clínico}

Paciente de sexo masculino de 57 años con antecedentes de cardiopatía coronaria, dislipidemia, hipertensión arterial, sobrepeso, infarto agudo al miocardio y cirugía de revascularización miocárdica en febrero del año 2008.

Consultó en el servicio de urgencias por un cuadro de dolor abdominal de menos de 24 horas de evolución, tipo cólico, irradiado a todo el abdomen. Asociado a náuseas y distensión abdominal leve, sin fiebre. Al examen físico presentaba un abdomen blando, depresible, sensible en forma difusa, más en fosa ilíaca derecha, sin signos de irritación peritoneal.

El estudio de laboratorio muestra: hemoglobina $16,3 \mathrm{gr} / \mathrm{L}$, hematocrito $46 \%$, leucocitos $10.850 / \mathrm{mm}^{2}$, proteína $C$ reactiva $1 \mathrm{mg} / \mathrm{L}$, creatinina $0,93 \mathrm{mg} / \mathrm{dL}$, bilirrubina total $1,18 \mathrm{mg} / \mathrm{dL}$, fosfatasa alcalina 89 UI/L, albúmina $4,7 \mathrm{gr} / \mathrm{dL}$ y proteínas totales $7,8 \mathrm{gr} /$ $\mathrm{dL}$.

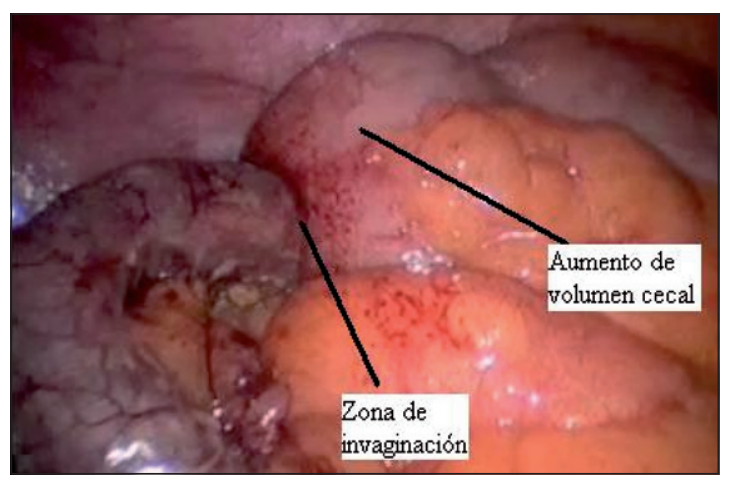

Figura 1. Se observa el apéndice cecal inflamado, con su base invaginada en el ciego, el que presenta un aspecto inflamatorio.

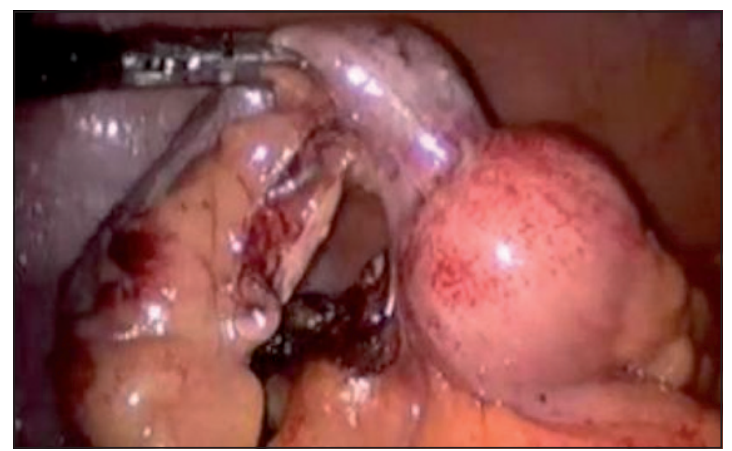

Figura 2. Liberación amplia de la base apendicular y el ciego.
Por la sospecha de un cuadro de apendicitis aguda se realizó una ecotomografía abdominal, la que mostró un apéndice cecal inflamado adosado a la pared anterior, de $12 \mathrm{~mm}$ de diámetro máximo, con varios coprolitos en su interior y un aumento de ecogenicidad de la grasa mesentérica a su alrededor.

El abordaje quirúrgico se realizó por vía laparoscópica. Se identificó el apéndice cecal flegmonoso en sus dos tercios distales, sin embargo, hacia la base se observó una imagen que impresionaba como una invaginación del segmento proximal del apéndice en la base del ciego, la que se encontraba eritematosa y aumentada de volumen (Figura 1). Se realizó una amplia liberación de la base y del polo cecal, que a la compresión impresionaba blando, no tumoral (Figura 2). Al tensar el apéndice se apreció con más claridad su relación con el ciego y la zona aumentada de volumen. Por ello se decidió completar el procedimiento por vía laparoscópica. Con un stappler lineal cortante se seccionó a nivel cecal (Figura 3), reforzando el área de sección con una sutura invaginante de vicryl 3-0. El estudio anatomopatológico informó un apéndice cecal de $7,5 \mathrm{~cm}$ de longitud y 1,5 de diámetro transverso con sutura metálica en el borde de sección cecal. Serosa café pardusca, opaca y discretamente distendida en el fondo. Mucosa congestiva y lumen sin contenido. Al examen microscópico se observaba la pared de arquitectura conservada con infiltrado inflamatorio polimorfonuclear, hemorragia y depósitos de fibrina. La inflamación se extendía al meso circundante, sin atipías. No se encontró fecalitos en el lumen apendicular. Conclusión: apendicitis aguda flegmonosa y peri-apendicitis aguda.

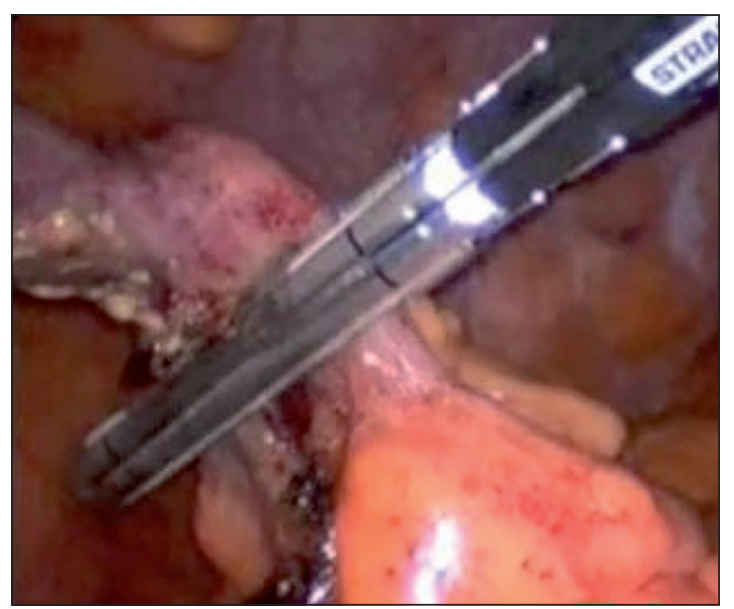

Figura 3. Sección con stapler a nivel cecal. 


\section{Discusión}

La invaginación apendicular fue descrita por primera vez por Mc Kidd el año $1858^{1}$. Es un cuadro muy poco frecuente, con una incidencia de $0,01 \%{ }^{2}$.

Se ha descrito más frecuentemente en niños y en personas de sexo masculino, el 50\% de los casos se presenta en menores de 10 años $^{3}$.

La invaginación del apéndice cecal se puede presentar en diversos grados. Mc-Swain, la clasificó anatómicamente de acuerdo al segmento invaginado dividiéndola a su vez en invaginación primaria y secundaria ${ }^{4}$. La forma de presentación clínica es muy variable y puede ir desde un caso de apendicitis aguda a un cuadro de intususcepción intestinal con síntomas obstructivos y rectorragia, sin embargo, también pueden ser asintomáticos y constituir un hallazgos en laparotomías diagnósticas o bien en una colonoscopia. La forma de presentación más frecuente es la descrita en este caso, la que corresponde a una invaginación parcial del apéndice en el ciego, con un cuadro de apendicitis aguda concomitante ${ }^{5}$. Pero, sin duda, el tipo de invaginación de más difícil diagnóstico es el tipo IV de Mc Swain ${ }^{4}$, que corresponde a una invaginación completa del apéndice cecal en el ciego. En estos casos se puede pasar por alto el diagnóstico asumiendo que el paciente tiene una ausencia congénita del apéndice. En la cirugía laparoscópica esto cobra mayor importancia ya que al no palpar el ciego aumenta la posibilidad de no diagnosticar una lesión de este tipo ${ }^{6}$.

El procedimiento más habitual en estos pacientes es la apendicectomía, sin embargo, algunos casos pueden llegar a requerir una hemicolectomía. El tratamiento va a depender del grado de compromiso apendicular y/o del ciego, de los factores asociados predisponentes y de la posibilidad de descartar una patología neoplásica durante el procedimiento quirúrgico.

\section{Conclusión}

La invaginación apendicular puede simular un tumor cecal, sin embargo, la exploración laparoscópica ayuda al reconocimiento visual. La disponibilidad de suturas mecánicas para uso laparoscópico ayuda a resolver con eficacia y seguridad este cuadro. Aunque es poco frecuente, la invaginación apendicular debe tenerse presente en el diagnóstico diferencial de aquellos pacientes con presentaciones atípicas, o bien en una laparoscopia diagnóstica en la que no se logra identificar el apéndice cecal.

\section{Referencias}

1. McKidd J. Case of invagination of cecum and appendix. Edinburgh Med J. 185;84:793.

2. Collins DC. Seventy-one thousand human appendix specimens. A final report summarizing forty years study. Am J Proctol. 1963;14:65-381.

3. Fournet-Fayard J, Louis D, Carlioz P, Tran-Minh V. Invagination primaire de l'appendice ileo-cecal. Pediatrie 1981;7:557-61.

4. McSwain B. Intussusception of the appendix. South Med J. 1941;34:263-71.

5. Lauwers GY, Predergast NC, Wahl SJ, Bagchi S. Invagination of vermiform appendix. Dig Dis Sci. 1993;38:565-8.

6. Vogelaar F, Molenaar I, Adhin S, Steenvoorde P. Invagination of the appendix: Diagnostic laparoscopy? Dig Dis Sci. 2004;49:351-2. 